\title{
Cognitive Style Differences in Attention Distribution Regarding Calligraphic Perception
}

This article was published in the following Dove Press journal:

Psychology Research and Behavior Management

\section{Tinghu Kang \\ Ping Wang \\ Hui Zhang}

Visual Cognition Laboratory, School of Psychology, Northwest Normal University, Lanzhou City, Gansu Province, People's Republic of China
Correspondence: Ping Wang Graduate Apartment, Northwest Normal University, Anning District, Lanzhou City, Gansu Province, People's Republic of China

Tel +86- 18893842473

Email137053602@qq.com
Purpose: Calligraphy is the most unique form of artistic expression in Chinese culture. However, most studies that used calligraphy as a research object only explored its artistic value from an artistic perspective. Thus, we know little about the information processing and influencing factors of calligraphic perception. Thus, we aimed to determine whether there are differences in attention distribution due to cognitive style in the process of calligraphic perception.

Methods: The calligraphy of Lan Ting $J i X u$, which is known as the first running script in the history of Chinese calligraphy, was selected as the experimental material. The study used eye movement experiments to explore the differences in cognitive styles of attention distribution when perceiving calligraphy, through the analysis of eye movement data of participants.

Results: The results showed that field-independent participants had more fixation duration, number of fixations, and saccade angle when they perceived calligraphic works than those who were field-dependent. In other words, field-independent individuals spend more attention resources in the perceptual process. In addition, through data analysis, it was found that fixation duration, number of fixations, and saccade angle in the middle position of calligraphy are larger than the data on both sides of the calligraphy. In other words, when individuals perceive calligraphy, the content in the middle position can attract more attention resources than those on both sides.

Conclusion: We found that individuals with different cognitive styles have differences in attention distribution in the process of perceiving calligraphy.

Keywords: Chinese characters, field-dependent, field-independent, fixation duration, saccade angles

\section{Introduction}

Chinese characters are traditional symbols of Chinese traditional culture. According to the records in Origin of Chinese Characters, ${ }^{1}$ the generation of Chinese characters has experienced three stages: image, borrowing, and echoism. Chinese characters have gradually evolved from pictures and symbols to creation and stereotypes, from ancient seal characters to small seal scripts, and eventually to official, regular, running, and cursive scripts that use different methods. The development of Chinese characters ranges from concrete to abstract_-from expressing meaning to expressing sound, and from complex to simple, using simpler symbols to express concrete and complex things.

The writing of Chinese characters is a unique art form called calligraphy. It has the duality of time and space aesthetics, which is based on words, and its 
aesthetic character has evolved from a preliminary development to a gradually enriched development process. $^{2}$ Running script is a type of calligraphy between regular and cursive script that appeared in the Han Dynasty, which tended to mature during the Jin Dynasty. In the Tang and Song Dynasties, it became increasingly popular and prosperous. ${ }^{3}$ As the foundation of calligraphy, running script is excellent in both practical life and lyrical art. It contains not only the rigor and regularity of a regular script, but also the flexibility and flow of the cursive script. Although the running script is written word by word at a time, they give people a sense of harmonious connection between words, which makes them feel that the calligraphy is integrity. The running script is simple and free - the words are flexible, natural, simple, easy to identify, and have a strong sense of rhythm. Therefore, running script has become the most commonly used form of calligraphy, with its popularity, duration, and convenience of use incomparable with others. $^{4}$

People's cognition and appreciation of artworks are related to their information processing characteristics; people tend to experience subjective reactions to their stylistic and structural properties when viewing them. ${ }^{5}$ In his study, Yarbus ${ }^{6}$ asked observers to freely examine different types of pictures. He recorded the observers' eye movement characteristics, and the results showed that their attention is usually held only by certain elements of the picture; they noticed different elements while appreciating the works, leading to different information from each work. Furthermore, because different items of information are usually localized in different parts of an object, the distribution of the points of fixation on an object vary correspondingly, depending on the character of the information that the subjects must obtain. Tatler, Wade, Kwan, Findlay, and Velichkovsky ${ }^{7}$ verified Yarbus' experiment and reached the same conclusion. Similarly, they found that the space allocation of attention changes with the appreciation of its purpose, and more attention is paid to the important part of the painting. Furthermore, in the field of art research, the influence of individual differences on art appreciation has always been an explored topic. Interestingness, ${ }^{8}$ emotional factors, ${ }^{9}$ social status, ${ }^{10}$ and cultural or economic background, in general, ${ }^{9}$ are known to influence aesthetic judgments. Savarese and Miller ${ }^{11}$ observed that greater field-independence tends to be associated with greater preference for painterly than linear art. As a unique form of artistic expression in China, calligraphy contains not only Chinese character art, but also linear art.

Since the 1950s, cognitive science has developed rapidly, emphasizing the cognitive value of factors such as feelings and emotions. At the same time, it is proposed that cognitive ability is realized on the basis of body-brain activities; the brain is malleable and will dynamically change according to changes in the situation. ${ }^{12}$ Cognition is perceptual in nature and shares system perception at the cognitive and neural levels. ${ }^{13}$ Evidence shows that the lateral prefrontal cortex (PFC) is related to cognitive control, ${ }^{14,15}$ and in the research of Cupchik, Vartanian, Crawley, and Mikulis, ${ }^{16}$ PFC appears to be a key structure in the orientation of cognitive and perceptual organization toward beauty in subjects without visual arts training. When we view visual images in our daily lives, our perception is toward object recognition. On the contrary, when we regard visual images as works of art, we also tend to have subjective reactions to their style and structural characteristics. ${ }^{5}$ Therefore, there are differences in the understanding of beauty among individuals. Cognitive style, also known as cognitive mode, ${ }^{16}$ is the first connection between cognition and style, and has been described as a person's typical or habitual mode of problem-solving, thinking, perceiving, and remembering. Cognitive style is an important individual difference variable; ${ }^{17}$ there are not only differences in individual behaviors, perceptions, and decisions, ${ }^{18,19}$ but also in individual cognitive processes, such as attention ${ }^{20}$ and memory, among others. During information processing, the ability of an individual to recognize stimuli from the background is different when it comes to cognitive style. Witkin and Goodenough ${ }^{21}$ believed that field-independence (FI) tends to rely on internal perceptual clues as guidance in the processing of information, while field-dependence (FD) tends to rely on external references or the external environment as clues. Wirth, Matthes, Schemer, and Kissling ${ }^{22}$ found that individuals with FI are more likely to identify stimulating objects from a vague environment and are less susceptible to environmental influences, while those with FD are the opposite. For example, it is important for the driver to identify potential hazards from the complex traffic environment. As a result, they can make defensive and protective measures against danger rapidly. Ernest, GhezelAyagh, and $\mathrm{Kush}^{23}$ believe that drivers with FI are better able to allocate attention resources to traffic information than those with FD, and have a better ability to monitor, extract, and organize information. The reaction in 
the eye movement index shows that drivers with FI have more fixations than those with FD. Cognitive style is an important variable of individual difference. The combination of different cognitive styles and thinking process determines the difference of individual creativity and aesthetics. $^{24}$

In recent years, research on cognitive styles has begun to integrate eye movement. Nisiforou and Laghos ${ }^{25}$ found that individuals with different cognitive styles have different eye movement patterns. For example, during the visual image search task, the scan paths of the gaze plots of users with FD were more disoriented and disorganized than the scan paths of those with FI. Eye movement recording technology is a research method that uses an eye movement recorder to take note and analyze individuals' eye movement indicators when reading, viewing pictures, and recognizing real scenes, so as to reveal their psychological processes and rules. ${ }^{26}$ Researchers can understand an individual's eye movement pattern through eye movement indicators to reflect the individual's cognitive process. For example, the number of fixations can reflect the cognitive processing load of individuals, and the greater the cognitive load, the greater the number of fixations. In addition, it can also show the regression-out reflecting the state of the individual's difficulty in processing in the early processing stage of interest area. ${ }^{27}$ Moreover, saccadic size can reflect the situation of individual information extraction. Generally, a large saccadic size has higher efficiency and indicates that individuals get more information at one time. ${ }^{28}$ Furthermore, visual search data reveal that when subjects engage in activities such as picture viewing, reading, or performing cognitive tasks, differences in eye movement patterns between individuals imply differences in attention, which reflects differences in cognitive functioning. ${ }^{29}$ Eye movement indicators can more intuitively reflect real-time attentional dynamic motion trajectories than behavioral data, such as reaction time or correct rate. ${ }^{30}$ Meanwhile, eye movement pattern can also record temporal and spatial information about attention selection during this process, which can further explain other psychological phenomena, such as working memory representations, ${ }^{31,32}$ attention conversion, ${ }^{33}$ and clue guiding effect, ${ }^{31}$ among others.

This study was based on the view of FI and FD; an eye tracker to record eye movement indicators of different cognitive styles when individuals perceive works of running script calligraphy was used to explore the differences in eye movement patterns and characteristics of attention distribution between FI and FD. The research hypothesis was that individuals with FI consume more attention resources in the process of perceiving works of calligraphy than those with FD. In addition, we hypothesized that there are differences in space of attention between them. No matter what cognitive style, individuals pay the most attention to the middle position of each work, which is not affected by the writing habits of calligraphy.

\section{Methods}

\section{Participants}

In order to investigate the attention distribution and cognitive processing characteristics in the perceptual process of calligraphy, the study applied the cognitive style scale and eye movement recorder to survey 50 college students recruited. According to the score division criteria of the Embedded Figures Test (EFT) revised by Beijing Normal University, 40 college students with typical cognitive style were selected to participate in this experiment. The FI group was 21 participants (8 males, 13 females, $M_{\text {age }} 24.21 \pm 2.42$ ), the EFT score was $46.22 \pm 1.91$; and the FD group was 19 participants (6 males, 13 females, $\left.M_{\text {age }} 24.24 \pm 2.74\right)$, the EFT score was $52.98 \pm 1.81$. Through independent sample $t$-test, there was a significant difference in cognitive style between the two groups, $t(38)=11.51, p<0.05$. All participants had normal or corrected-to-normal vision, without color blindness or color weakness. And there provided their informed consent before the experiment start, received payment upon completion, and were naive to the underlying purposes of the experiment.

\section{Research Design}

The experiment was conducted using a 2 (cognitive style: FI, FD) $\times 5$ (interest area: 1-5) factorial design. The area of interest (AOI) of the calligraphy was divided into five levels according to the number of columns, and from right to left; the interest areas were one to five, as shown in Figure 1. Eye movement indicators recorded by the eye tracker, including fixation duration, number of fixations, and saccade angle were the dependent variables.

Fixation duration, also known as total dwell time, ${ }^{34}$ total reading time ${ }^{35}$ or total viewing time, ${ }^{36}$ is the sum of the time of all fixation points in the area of interest (AOI), reflects the processing time of the area. ${ }^{28}$ The longer the fixation time, the more interested the individual is in the region. ${ }^{37}$ The number of fixations is the total number of 


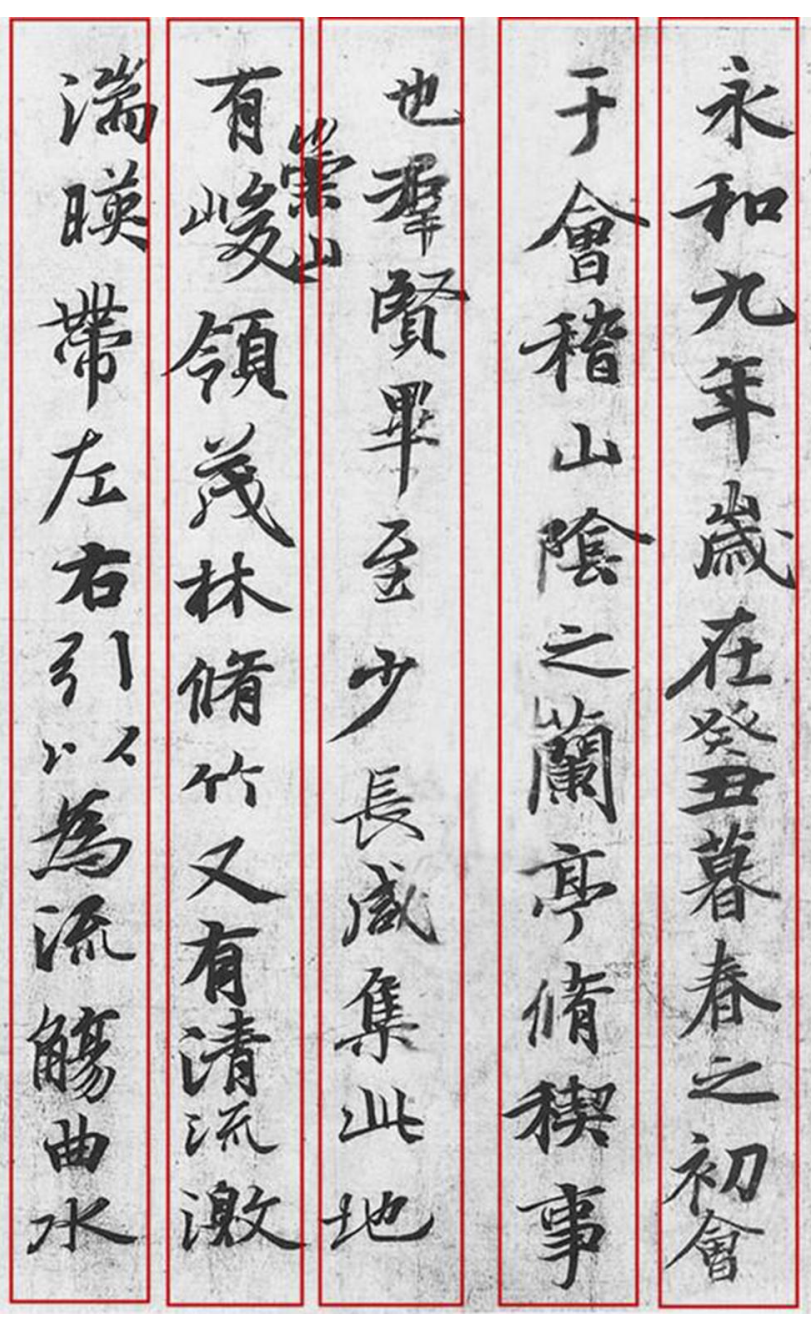

Figure I The five interest areas of calligraphy.

fixations of an AOI. ${ }^{27}$ The number of fixations can reflect the cognitive processing load of individuals, and the greater the cognitive load, the greater the number of fixations. ${ }^{26}$ In addition, it can also show the regressionout reflecting the state of the individual's difficulty in processing in the early processing stage of interest area. ${ }^{27}$ Moreover, saccadic size can reflect the situation of individual information extraction. Generally, a large saccadic size has higher efficiency, and indicates that individuals get more information at one time. ${ }^{28}$ In a series of studies by Irwin et al, although the coding of visual stimuli is inhibited during saccade, the cognitive processing of vocabulary is not inhibited. ${ }^{38-40}$

\section{Material}

\section{Calligraphy}

The official experimental materials were selected from the running script calligraphy prologue of Lan Ting $\mathrm{Ji} \mathrm{Xu}$
(Figure 1). The copybook was cropped using Photoshop software. After cropping, each picture presented five columns of calligraphy content, with each picture having a pixel size of $1024 \times 566$. Unified post-processing of each picture was done and stamp marks on the pictures were removed as they affect participants' perception of the content of the calligraphy materials.

\section{Cognitive Style Scale}

The cognitive style assessment tool used in this study is the Embedded Figures Test (EFT), which is revised by the Department of Psychology of Beijing Normal University. ${ }^{41}$ There are three sections with 9,10 , and 10 items, respectively, and the number of simple figures correctly identified in the last two sections constitutes the raw score. The participant's task is to outline a simple figure in a complex pattern. ${ }^{42}$ There are nine simple figures; in each part, participants were required to find simple graphics that are hidden in the complex pattern within the specified time and mark them with a pen. The simple graphics have specific designations, and their corresponding ordinal number to be found is marked under each complex pattern. According to the norm, the adult group's time limit for each part is $6 \mathrm{~min}$, with a total of $12 \mathrm{~min}^{43}$ The EFT reliability coefficient is 0.90 .

The test score is obtained by adding the second and third parts, with one point for each question, resulting in a maximum score of 20 points. If the direction, size, and scale of the graphic described by the tester do not match the simple graphic, no score will be given. ${ }^{43}$ Finally, according to the adult norm for the test, the following calculation formula was used: $t=$ (statistical score norm score $) \div$ norm standard deviation, $T=t \times(10+$ 50 ), to obtain the score of each tester. $T$ is the final statistical data; if $T$ is greater than 50 , it indicates that the test takers tend to be with FI, and they tend to be with FD if it is less than $50{ }^{44}$

\section{Instrument}

Monocular eye data were recorded using an EyeLink 1000 Plus Tower Mount eye tracker (SR Research, Ontario, Canada) with a temporal resolution set to $2000 \mathrm{~Hz}$. For calibration, validation, drift correction, and computation of the eye movement parameters (blinks, fixations, saccades), we used the manufacturer's software. ${ }^{45}$ The Experiment Builder software ${ }^{33}$ was used for stimulus presentation and response recording. The velocity threshold for saccade detection was $35^{\circ} / \mathrm{s}$, while the acceleration threshold was 
$9500 \%$ s. A 9-point calibration procedure was performed before each block as well as a drift correction before each trial. The spatial resolution was typically better than $0.01^{\circ}$. Participants' heads were stabilized using the chin and forehead rest of the EyeLink Tower Mount (SR Research, Ontario, Canada), and were placed $65 \mathrm{~cm}$ away from the screen. Stimuli were presented on a 19-in LG flatron L1920P monitor run at $60 \mathrm{~Hz}$ with $1240 \times 1024$ pixel resolution, and the tracking range was $60^{\circ}$ vertical $40^{\circ}$.

\section{Procedure}

The experiment was conducted in a dedicated eye movement laboratory. In each test, participants responded independently in a quiet environment. Before starting the experiment, participants completed the informed consent and basic information forms. Participants were asked to put their chin on the chin bracket and keep their posture as constant as possible, and a 9-point calibration procedure was performed to map eye position to screen coordinates. Participants were told that a piece of calligraphy will be displayed on the screen, and were asked to press any key when they are finished appreciating it. During this time, they were instructed to view each picture as if they were appreciating artworks in a gallery. Given the free viewing nature of this task, we did not require participants to provide any responses.

Each participant viewed all stimuli (23 pictures). Pictures were presented in the same order for each participant. Finally, participants completed the cognitive style test independently after the formal eye movement experiment.

\section{Data Processing}

The data analysis software was built into the Eyelink 1000 Plus eye tracker to export data, and preliminary sorting and screening of data were conducted using Microsoft Excel. The eye movement data were matched with the basic information and cognitive style scores, and were entered into the same table We used SPSS ( $\mathrm{v}$ 20.0; China) for the statistical processing of data.

\section{Results}

The eye tracker automatically recorded and collected the eye movement data of the participants while they were observing the pictures. We eliminated $1 \%$ invalid data due to subjects' head movement or tired eyes during the experiment, since these factors may lead to inaccurate recording of data. The remaining valid data were then processed and exported, and the resulting eye movement data were statistically analyzed. We mainly used one-way ANOVA of eye movement data of the different cognitive styles in the AOI of the pictures. The main test data were fixation duration, number of fixations, and saccade angle.

\section{Fixation Duration}

The descriptive statistical results of the fixation duration of participants with two different cognitive styles in different AOIs are shown in Table 1.

According to the one-way ANOVA, there are significant differences in cognitive style and AOI. The results showed that the $F$ of the main effect of participants' factors between cognitive style and AOI on fixation duration was significant, but the interaction was not significant, $F(1,39)=0.670, p>$ $0.05, \eta^{2}=0.014$. There were significant differences between different cognitive styles, $F(1)=4.689, p<0.05, \eta^{2}=0.024$, and fixation duration of those with FI $(M=0.186, S D=0.080)$ was longer than those with FD $(M=0.166, S D=0.102)$. There were differences between different AOIs, $F(39)=14.606$, $p<0.05, \eta^{2}=0.235$, and the AOI of the middle position is longer than that of both sides.

\section{Number of Fixations}

The descriptive statistical results of the number of fixations of participants with two different cognitive styles in different AOIs are shown in Table 2.

The lower the number of fixations, the more selective the fixation area is, and the more information a single fixation can obtain. According to the one-way ANOVA, there are significant differences in cognitive style and AOI. The results showed that the $F$ of the main effect of

Table I Average Fixation Duration (s) of Different Cognitive Styles in Different AOI

\begin{tabular}{|l|c|c|c|c|c|c|c|c|c|c|c|c|}
\hline & \multicolumn{2}{|c|}{ Sum } & \multicolumn{2}{|c|}{ I } & \multicolumn{2}{c|}{2} & \multicolumn{2}{c|}{3} & \multicolumn{2}{c|}{4} & \multicolumn{2}{c|}{5} \\
\cline { 2 - 13 } & $M$ & SD & $M$ & SD & $M$ & SD & $M$ & SD & $M$ & SD & $M$ & SD \\
\hline FI & 0.186 & 0.080 & 0.144 & 0.074 & 0.200 & 0.045 & 0.216 & 0.050 & 0.244 & 0.087 & 0.127 & 0.080 \\
FD & 0.166 & 0.102 & 0.136 & 0.089 & 0.194 & 0.088 & 0.210 & 0.099 & 0.195 & 0.117 & 0.090 & 0.068 \\
\hline
\end{tabular}

Abbreviation: $\mathrm{AOI}$, area of interest. 
Table 2 Average Number of Fixations of Different Cognitive Styles in Different AOI

\begin{tabular}{|l|c|c|c|c|c|c|c|c|c|c|c|c|}
\hline \multirow{2}{*}{} & \multicolumn{2}{|c|}{ Sum } & \multicolumn{2}{|c|}{ I } & \multicolumn{2}{c|}{2} & \multicolumn{2}{c|}{3} & \multicolumn{2}{c|}{4} & \multicolumn{2}{c|}{5} \\
\cline { 2 - 13 } & $M$ & SD & $M$ & SD & $M$ & SD & $M$ & SD & $M$ & SD & M & SD \\
\hline FI & 9.952 & 6.024 & 8.447 & 6.402 & 10.509 & 6.047 & 10.460 & 5.561 & 12.833 & 6.213 & 7.510 & 4.864 \\
FD & 7.586 & 5.920 & 6.713 & 5.578 & 8.500 & 6.626 & 8.966 & 5.819 & 8.890 & 6.526 & 4.621 & 3.948 \\
\hline
\end{tabular}

participants' factors between cognitive style and AOI on the number of fixations is significant, but the interaction is not significant, $F(1,39)=0.287, p>0.05, \eta^{2}=0.006$. There were significant differences between different cognitive styles, $F(1,39)=9.936, p<0.05, \eta^{2}=0.050$, and the number of fixations of those with FI $(M=9.952$, $S D=6.024)$ is longer than those with $\mathrm{FD}(M=7.586$, $S D=5.920)$. There are also differences between different AOIs, $F(1,39)=4.527, p<0.05, \eta^{2}=0.087$, and the AOI of the middle position is higher than that of both sides.

\section{Saccade Angle}

The descriptive statistical results of the saccade angle of participants with two different cognitive styles in different AOIs are shown in Table 3.

According to the one-

way ANOVA, there are significant differences in cognitive style and AOI. The results showed that the $F$ of the main effect of participants' factors between cognitive style and AOI on saccade angle was significant, but the interaction was not significant, $F(1,39)=1.567, p>0.05, \eta^{2}=0.032$. There were significant differences between different cognitive styles, $F(1,39)=4.799, p<0.05, \eta^{2}=0.025$, and saccade angle of those with FI $(M=15.304, S D=48.296)$ is longer than those with FD $(M=0.171, S D=56.818)$. There are also differences between different AOIs, $F(1,39)=$ 3.399, $p<0.05, \eta^{2}=0.068$, and the AOI of the middle position is larger than that of both sides.

\section{Discussion}

The perceptual process of calligraphy is actually a process of aesthetic psychological activity. From a psychological point of view, this perceptual process is a whole organic system that contains emotion, attention, imagination, association, feeling, understanding, and other psychological factors. Calligraphic perception does not involve a mere glimpse at the calligraphic work, but the appreciation of every stroke, word, and column. In the system of calligraphic perception, psychological factors are independent and interrelated as well as complement each other, so that the perceptual process of calligraphy becomes hierarchical and continuous. ${ }^{46}$ Hence, this study used the method of eye movement recording to understand whether there are differences in calligraphic perception among individuals with different cognitive styles, and determined how to respond to eye movement indicators if such differences exist. At the same time, when perceiving calligraphy, we aimed to identify the perceptual process of individuals and whether different positions affect their calligraphic perception. The study found that in the process of perceiving running script, participants with FI had longer fixation durations, more fixation numbers, and larger saccade angles than those with FD. In addition, the central position of calligraphy has more fixation duration and fixation number than the two sides, but the saccade angle is smaller.

\section{Attention to the Distribution of Resources in the Perceptual Process of Calligraphy}

In this study, it was found that when perceiving calligraphy, there are differences in the eye movement patterns between participants with FI and those with FD. Specifically, participants with FI have significantly longer fixation duration, more fixation number, and larger saccade

Table 3 Average Number of Saccade Angle of Different Cognitive Styles in Different AOI

\begin{tabular}{|l|c|c|c|c|c|c|c|c|c|c|c|c|}
\hline \multirow{2}{*}{} & \multicolumn{2}{|c|}{ Sum } & \multicolumn{2}{c|}{ I } & \multicolumn{2}{c|}{ 2 } & \multicolumn{2}{c|}{3} & \multicolumn{2}{|c|}{4} & \multicolumn{2}{c|}{5} \\
\cline { 2 - 13 } & M & SD & $M$ & SD & M & SD & M & SD & M & SD & M & SD \\
\hline FI & 15.304 & 48.296 & 12.409 & 29.134 & 25.365 & 41.878 & 9.251 & 61.253 & 23.278 & 56.335 & 6.214 & 47.895 \\
FD & 0.171 & 56.818 & 17.478 & 33.092 & 19.807 & 39.731 & 2.039 & 67.601 & -1.431 & 60.758 & -41.309 & 60.791 \\
\hline
\end{tabular}


angle than those with FD. This shows that, compared to participants with FD, participants with FI have a deeper perception of the whole work and higher processing efficiency when perceiving calligraphy.

Goode, Goddard, and Pascual-Leone ${ }^{47}$ found that when working memory is applied to complex materials, analytic individuals invest more and better distribute attention resources to actively carrying out deeper information processing than holist individuals. However, this also leads to analytic individuals being more affected by interference stimuli. In case of limited attention resources, analytic individuals are more likely to "divide" attention resources in advance according to object characteristics or spatial location, which means that there is a difficulty in focusing the attention resources that were previously distributed to each part of the target stimulus in various parts because of the limited attention resources as a whole. As for holist individuals, attention resources are allocated in a "holistic" way; therefore, when attention resources are limited, most are focused on the target stimulus first. ${ }^{48}$ In other words, individuals with FI tend to regard objects as independent and separate components. Hence, when processing a calligraphic work, participants with FI regard it separately and in parts, and they need to process each part one by one as well as invest more attention resources to extract useful information from it in case of calligraphic perception. Thus, participants with FI have more fixation duration and greater fixation number in the process of perceiving calligraphy. On the contrary, individuals with FD tend to regard perceptual objects as a complete and unified whole, and rely on external reference or the external environment for clues in information processing. Therefore, participants with FD have shorter fixation duration and less number of fixations when perceiving calligraphy.

At the same time, individuals with FI usually rely on internal perceptual clues for guidance in information processing, so memory clues are not set up and there is no excessive connection between the various information; therefore, the integration process of calligraphy is slow and cumbersome. However, individuals with FD rely on external references or the external environment for clues in information processing, and perceive calligraphy from a holistic perspective, which makes it easier to integrate content. Moreover, they recall more social information than individuals with FI. ${ }^{49}$ Calligraphy, as a type of art with social characteristics, ${ }^{50}$ contains not only the noble character of calligraphers of past dynasties but also the unique elegant connotation of Chinese traditional culture. Aesthetics is closely related to the human body, the physiological sensation caused by the object itself and the internal memory. ${ }^{51}$ Therefore, in the perceptual process of calligraphy, participants with FD could recall the information they had previously noticed better than those with FI, resulting to less fixation duration and fixation number in calligraphic perception. Meanwhile, the saccade angle reflects the perceptual span of the individuals to a certain extent, and a larger saccade angle indicates a larger amount of information acquired at once. ${ }^{28}$ Participants with FD need to pay more attention to process calligraphy; however, they can obtain more information at one time, and their processing efficiency is higher.

In addition, cognitive style is related to lateralization of the brain - individuals with FD tend to lateralize the right hemisphere, while those with FI tend to lateralize the left. $^{52}$ There are also obvious similarities between the function of the brain's frontal lobe and cognitive style; cognitive processes that are related to the frontal lobe may affect the characteristics of sensory information, which is used for cognitive processing. Meanwhile, cognitive processes that are related to only one hemisphere may selectively participate in processing. In the field of visual perception, when performing a simple and random scanning task, such as free viewing of pictures, sensory information has a relatively low structure and is mainly involved in the processing of the right hemisphere. In other words, individuals with FD who tend to lateralize the right hemisphere will perform better in free viewing of pictures task.

\section{Positional Features in the Perceptual Process of Calligraphy}

This study found differences in AOIs between participants with FI and FD with regard to calligraphic perception. Specifically, in the second, third, and fourth AOI, the fixation duration and fixation number of participants are longer and greater, respectively, than that in the other two AOIs. However, in the third AOI, the saccade angle of participants is significantly smaller than that in the other four AOIs. In any AOI, the fixation duration, number of fixations, and saccade angle of participants with FI are greater than that of those with FD.

In the same time period, participants observed the main body in the middle of the screen the most. Almost all participants paid attention to the middle position of the 
screen at the first instance; that is to say, participants have a deeper level of information processing in the middle position. ${ }^{53}$ In calligraphy, the second, third, and fourth AOIs are all located in the middle of the work. Therefore, in the process of perception of participants, the contents of the three AOIs are perceived the most times, leading to a significantly greater fixation duration and number of fixations than those of the remaining two AOIs on both sides. Yarbus ${ }^{6}$ also pointed out that the middle part of the screen attracts more attention. In this study, we determined whether participants would pay more attention to the distribution of resources in the middle of the work when perceiving calligraphy. As a result, we found that when participants perceive the content in the middle position, they pay less attention to the content on both sides; hence, the third AOI had the smallest saccade angle among the five AOIs.

In this study, the fixation duration and number of fixations in the second, third, and fourth AOIs are larger. However, in the third AOI, the saccade angle is significantly smaller than the other AOIs. To a certain extent, this conclusion also confirms the finding that the content located in the middle of the screen is perceived the most times, and that the processing level of the content information here is deeper.

Art is one of the ways of human thinking and emotional expression. It is based on perceptual, leaping, and inspired thinking, which is different from the thinking of other disciplines. Individuals are influenced by cognitive style to understand and perceive artistic works. The results of this study confirm that individuals' perception process of calligraphy art is influenced by cognitive style. As an important source of cognitive activities of FD individuals, external information has important existential value for their artistic creation. ${ }^{54}$ The external resources available to the FD individual are more abundant, and the internal emotional activities are more sensitive and delicate. In contrast, FI is more adept at using internal clues to complete information processing $^{55}$ and tend to conduct internal construction in artistic creation. In the future, both calligraphy appreciation and calligraphy creation can be considered from the perspective of individual cognitive characteristics, so as to better appreciate calligraphy and create calligraphy that are more acceptable and understood by the public.

\section{Limitations}

There are many pictures in this experiment but there is limited time and energy; hence, when selecting eye movement indicators, only the most representative was used for discussion. However, it is undoubtedly true that the unused indicators have great research value. In addition, this paper only explored the reasons why subjects with different cognitive styles show significant differences in perceiving running scripts and did not further consider deeper causal connections. Although the individual's cognition will affect his perceptual process of running calligraphy, it may not be the main influencing factor. Therefore, it is necessary to further explore other factors influencing individual perception of calligraphy.

Moreover, this study simply considered calligraphy as a kind of artistic work without taking into account its literary value and discussing whether the connotation of literature has an impact on calligraphic perception. Lastly, perceptual objects are only limited to works of running calligraphy and are not extended to other types of calligraphy.

\section{Conclusion}

This study found that the cognitive style and AOI have a significant influence on calligraphic perception. Moreover, individuals with different cognitive styles have different eye movement patterns in observing calligraphy. However, this does not mean that a greater eye movement index value, such as fixation duration and saccade angle, leads to better calligraphic perception.

Only from the perspective of artistic appreciation of calligraphy, the results of the previous analysis show that participants with FI are more likely to distribute attention resources, which leads to a more efficient yet more likely to be disturbed calligraphic processing. Moreover, participants with FI find it may harder to recall previously perceived calligraphy content than those with FD, leading to difficulties in integrating the ongoing processing with the previously processed content. ${ }^{49}$ These reasons also lead to participants paying more attention to the perceptual process of calligraphy as well as greater fixation numbers and saccade angles. Because the content of the middle position attracts more attention, it also makes the fixation duration longer, the number of fixations greater, and the saccade angle smaller in the second, third, and fourth AOIs; this is not affected by the differences in writing habits of calligraphy.

\section{Ethics Approval and Informed Consent}

This study has been approved by the scientific and research Ethics Committee of the School of Psychology, 
Northwest Normal University, and has been approved by the Science and Research Ethics Committee. The study has been agreed by all participants and complies with the guidelines outlined in the Declaration of Helsinki.

\section{Disclosure}

The authors report no conflicts of interest in this work.

\section{References}

1. Shen X. Origin of Chinese Characters. Shanghai Ancient Books Publishing House; 1981.

2. Chen H. The cognition and development of traditional calligraphy art. J Shandong Univ. 2004;(3):120-125.

3. Liu R. Understanding of calligraphy in running script. Chin Calligraphy News. 2018;(2)

4. Shen S. On the mainstream status of running script in contemporary calligraphy. J Huzhou Teachers Coll. 1995;(4):70-75.

5. Cupchik GC, Vartanian O, Crawley A, Mikulis DJ. Viewing artworks: contributions of cognitive control and perceptual facilitation to aesthetic experience. Brain Cogn. 2009;70(1):84-91. doi:10.1016/ j.bandc.2009.01.003

6. Yarbus AL. Eye Movements and Vision. New York: Plenum Press; 1967.

7. Tatler BW, Wade NJ, Kwan H, Findlay JM, Velichkovsky BM. Yarbus, eye movements, and vision. IPerception. 2010;1(1):7-27. doi:10.1068/i0382

8. Berlyne DE. Studies in the new experimental aesthetics: steps toward an objective psychology of aesthetic appreciation. Hemisphere. 1974

9. Konecni VJ. Determinants of aesthetic preference and effects of exposure to aesthetic stimuli: social emotional, and cognitive factors. Prog Exp Pers Res. 1979;9:149-197. doi:10.1111/j.17496632.2009.04555.x

10. Ritterfeld U. Social heuristics in interior design preferences. J Environ Psychol. 2002;22(4):369-386. doi:10.1006/jevp.2002.0276

11. Savarese JM, Miller RJ. Artistic preferences and cognitive: perceptual style. Stud Art Educ. 1979;20(2):45. doi:10.2307/1319541

12. Barsalou LW. Grounded cognition. Annu Rev Psychol. 2008;59 (1):617-645. doi:10.1146/annurev.psych.59.103006.093639

13. Barsalou LW. Perceptual symbol systems. Behav Brain Sci. 1999;22 (4):577-660. doi:10.1017/s0140525x99002149

14. Burgess PW, Dumontheil I, Gilbert SJ. The gateway hypothesis of rostral prefrontal cortex (area 10) function. Trends Cogn Sci. 2007;11 (7):290-298. doi:10.1016/j.tics.2007.05.004

15. Ridderinkhof KR, Ullsperger M, Crone EA, Nieuwenhuis S. The role of the medial frontal cortex in cognitive control. Science. 2004;306 (5695):443-447. doi:10.1126/science. 1100301

16. Allport GW. Personality: a psychological interpretation. Am J Sociol. 1937;45(1):48-50.

17. Yang Z, Guo L. Research progress on cognitive style. Psychol Sci. 2001;24(3):326-329.

18. Armstrong SJ, Mahmud A. Experiential learning and the acquisition of managerial tacit knowledge. Acad Manag Learn Educ. 2008;7 (2):189-208. doi:10.5465/amle.2008.32712617

19. Mumford MD, Licuanan B. Leading for innovation: conclusions, issues, and directions. Leadersh Q. 2004;15(1):171. doi:10.1016/j. leaqua.2003.12.010

20. Guisande MA, Páramo MF, Tinajero C, Almeida LS. Field dependence-independence (FDI) cognitive style: an analysis of attentional functioning. Psicothema. 2007;19(4):572-577.

21. Witkin HA, Goodenough DR. Cognitive styles: essence and origins. Field dependence and field independence. Psychol Issues. 1981; 51:1-141.
22. Wirth W, Matthes J, Schemer C, Kissling AK. I see what you don't see the role of individual differences in field dependenceindependence as a predictor of product placement recall and brand liking. J Advert. 2011;40(4):85-99.

23. Ernest JB, GhezelAyagh H, Kush AK. Dynamic simulation of a direct carbonate fuel cell power plant. Fuel Cell Semin Organ Committee. 1996. doi: $10.2172 / 460168$

24. Yu G, Hou R. Question asking, cognitive style and creativity cultivation in school education. Educ Sci. 2012;20(4):54-58.

25. Nisiforou E, Laghos A. Field dependence-independence and eye movement patterns: investigating users' differences through an eye tracking study. Interact Comput. 2015;28(4):407-420. doi:10.1093/iwc/iwv015

26. Jin X. Understanding of the psychological significance of eye movement research methods. Chin Outside Sch Educ. 2012;(12):94.

27. Yan G, Xiong J, Zang C, Yu L, Cui L, Bai X. Review of major eye movement indicators in reading research. Adv Psychol Sci. 2013;21 (4):589-605. doi:10.3724/SP.J.1042.2013.00589

28. Zhang X, Ye W. Review of eye movement indicators in current reading research. Psychol Behav Res. 2006;4(3):236-240.

29. Koga K. Intercultural experiments as a research tool in the study of cognitive skill acquisition: Japanese character recognition and eye movements in non-Japanese subjects. Adv Psychol. 1989;58:279-291.

30. Geyer T, Von Mühlenen A, Müller HJ. What do eye movements reveal about the role of memory in visual search? $Q J$ Exp Psychol (Hove). 2007;60(7):924-935. doi:10.1080/17470210600831119

31. Soto D, Heinke D, Humphreys GW, Blanco MJ. Early, involuntary top-down guidance of attention from working memory. J Exp Psychol Hum Percept Perform. 2005;31(2):248-261. doi:10.1037/0096-152 3.31.2.248

32. Soto D, Humphreys GW, Heinke D. Working memory can guide pop-out search. Vision Res. 2006;46(6-7):1010-1018. doi:10.1016/j. visres.2005.09.008

33. Meyer K, Rasch T, Schnotz W. Effects of animation's speed of presentation on perceptual processing and learning. Learn Instr. 2010;20(2):136-145. doi:10.1016/j.learninstruc.2009.02.016

34. Holmqvist K, Nyström N, Andersson R, Dewhurst R, Jarodzka H, Van de Weijer J. Eye Tracking: A Comprehensive Guide to Methods and Measures. Oxford; 2011.

35. Clifton C, Staub A, Rayner K. Eye movements in reading words and sentences. Eye Movements Reading Words Sentences. 2007.

36. Inhoff AW, Radach R. Definition and Computation of Oculomotor Measures in the Study of Cognitive Processes. Eye Guidance Reading Scene Percept. 1998.

37. He $Z$ The effect of different BMI on food cue attentional bias under emotional induction (Doctoral dissertation). 2019.

38. Irwin JD. Lexical processing during saccadic eye movements. Cogn Psychol. 1998;36(1):1-27. doi:10.1006/cogp.1998.0682

39. Irwin DE, Brockmole JR. Suppressing where but not what: the effect of saccades on dorsal- and ventral-stream visual processing. Psychol Sci. 2010;15(7):467-473. doi:10.1111/j.0956-7976.2004.00703.x

40. Irwin D, Carlson-Radvansky E, Laura A. Cognitive suppression during saccadic eye movements. Psychol Sci (Wiley-Blackwell). 1996;7:83-88. doi:10.1111/j.1467-9280.1996.tb00334.x

41. Zhang H, Zheng R, Song H. Instructions for the Graphical Test of Cognitive Modes. 1981

42. Kepner MD, Neimark ED. Test-retest reliability and differential patterns of score change on the group embedded figures test. J Pers Soc Psychol. 1984;46(6):1405-1413. doi:10.1037/0022-3514.46.6.1405

43. Wang Y, Ouyang L. The effect of different cognitive styles of college students on the level of figure reasoning - also on the role of personality in the cognitive process. Psychol Sci. 2004;(2). doi:10.3969/j. issn.1671-6981.2004.02.041

44. Zhao N, Yang H. Experimental study on field independence-dependency characteristics of cognitive style of college students. Sports Sci Technol. 2016;(5). doi:10.14038/j.cnki.tykj.2016. 05.020 
45. SR Research Ltd. Data Viewer: 2014.

46. Zhu X. Psychological analysis in the process of calligraphy appreciation. Grand View of Fine Arts. 2010;(9):226-227. doi:10. 3969/j.issn.1002-2953.2010.09.119

47. Goode PE, Goddard PH, Pascual-Leone J. Event-related potentials index cognitive style differences during a serial-order recall task. Int J Psychophysiol. 2002;43(2):0-140. doi:10.1016/S0167-8760(01) 00158-1

48. Liang J. The Influence of Cognitive Style on Cognitive Processing (Doctoral Dissertation). Zhejiang University; 2012.

49. Li S. Research on the Brain Mechanism of Field-Dependent and Field-Independent Individuals in the Process of Information Processing (Doctoral Dissertation). Shandong Normal University; 2006.

50. Xie H. The cultural, human, social and international nature of Chinese calligraphy. China Dev. 2011;11(6):78-82. doi:10.3969/j. issn.1671-2404.2011.06.014
51. Wang S. The brain of the museum: the theory and application of embodied cognition in multi-sensory aesthetic perception. Museum Res. 2016;(4):12-22.

52. Cai L. Eye Movement Study on Visual Processing of Individuals with Different Field Cognition Styles (Doctoral Dissertation). Hunan Normal University; 2012.

53. Cao W, Che X, Qi L, Liu H. Eye movement experiment of the main position layout of the picture and its enlightenment to the construction of network video resources. J Dist Educ. 2013;(5). doi:10.3969/j. issn.1672-0008.2013.05.013

54. Cheng $\mathrm{L}, \mathrm{Hu} \mathrm{W}$, Jia X. The influence of cognitive inhibition on artistic creativity: the moderating effect of cognitive style. Psychol Dev Educ. 2015;(3):287-295.

55. Song G, Han S. Field dependence - a comparison of interference suppression in independent cognitive mode. Psychol Behav Stud. 2007;5(2):100-104.
Psychology Research and Behavior Management

\section{Publish your work in this journal}

Psychology Research and Behavior Management is an international, peer-reviewed, open access journal focusing on the science of psychology and its application in behavior management to develop improved outcomes in the clinical, educational, sports and business arenas. Specific topics covered in the journal include: Neuroscience, memory and decision making; Behavior modification and management; Clinical
Dovepress

applications; Business and sports performance management; Social and developmental studies; Animal studies. The manuscript management system is completely online and includes a very quick and fair peer-review system, which is all easy to use. Visit http://www. dovepress.com/testimonials.php to read real quotes from published authors. 